\title{
FREQUENCY DEPENDENCE OF THE POLARIZABILITY AND SUSCEPTIBILITY OF A CIRCULAR HOLE IN A THICK CONDUCTING WALL*
}

\author{
Wen-Hao Cheng, Alexei V. Fedotov and Robert L. Gluckstern \\ Physics Department, University of Maryland, College Park, MD 20742, USA
}

\begin{abstract}
We calculate a generalized polarizability and susceptibility of a circular hole in a thick metallic plate as a function of hole dimensions and wavelength. In particular, we construct a variational form which allows us to obtain accurate numerical results with a minimum of computational effort. Numerical results are obtained for a variety of hole dimensions relative to the wavelength. In addition, analytic results are obtained and shown to be accurate to second order in the ratio of the hole dimension to the wavelength for a vanishingly thin wall.
\end{abstract}

\section{INTRODUCTION}

The penetration of electric and magnetic ${ }^{\circledR e l d s ~ t h r o u g h ~ a ~ h o l e ~}$ in a metallic wall plays an important role in many devices. In an accelerator, such holes in the beam pipe serve to allow access for pumping, devices for beam current and beam position measurement, coupling between cavities, etc. In much of the early work the hole dimensions were considered to be very small compared to the wavelength. The purpose of this paper is to extend the calculation to include the effects of $\circledR$ nite wavelength, although we still con ${ }^{\circledR}$ ne our attention to wavelengths no smaller than the hole dimensions.

We rede ${ }^{\circledR}$ ne the conventional static treatment of polarizability and susceptibility in terms of the cavity detuning, deßning a new generalized polarizability and susceptibility. In this way, we include the frequency dependence of the polarizability and susceptibility as well as the contributions of higher multipole moments of the hole. But these generalized polarizability and susceptibilites should only be seen as intermediate vehicles to relate the coupling integrals of interest to the detuning of the cavity by the hole. We will obtain an expression for the detuning of the modes of the symmetric cavity structure due to the presence of the hole. The symmetric cavity structure consists of two identical cavities, each of the length $L$ and radius $b$. Clearly the modes will be either symmetric or antisymmetric in the axial coordinate. Our analysis will be limited to the modes near the $\mathrm{TM}_{0 \mathrm{~N} \ell}$, $\mathrm{TM}_{1 \mathrm{~N} \ell}$ and $\mathrm{TE}_{1 \mathrm{~N} \ell}$ modes of the unperturbed pillbox.

\section{GENERAL ANALYSIS}

Our analysis can be generalized to include cavity regions and iris regions of arbitrary cross section. Taking $z_{1}=0$ to be the left end of the left cavity, we can write the transverse electric ${ }^{\circledR}$ eld as

$$
\boldsymbol{E}_{\perp}^{(C)}\left(\boldsymbol{r}, z_{1}\right)=\sum_{n} a_{n} \boldsymbol{e}_{n}(\boldsymbol{r}) \frac{\sin \beta_{n} z_{1}}{\sin \beta_{n} L}
$$

\footnotetext{
*Work supported by the U.S. Department of Energy
}

where $\boldsymbol{r}$ stands for the transverse coordinates $x$ and $y$, and where the modes $\boldsymbol{e}_{n}$ may be either TM or TE. Here $\beta_{n}^{2}=k^{2}-$ $\gamma_{n}^{2}$, where $\gamma_{n}^{2}$ are the eigenvalues of the two dimensional scalar Helmholtz equation in the cavity region with the appropriate boundary conditions. We use Latin subscripts $(n, k m, \ldots)$ for the cavity region, and $k c / 2 \pi$ is the frequency.

The transverse electric ${ }^{\circledR}$ eld in the iris region can similarly be written as

$$
\boldsymbol{E}_{\perp}^{(I)}\left(\boldsymbol{r}, z_{2}\right)=\sum_{\nu} b_{\nu} \boldsymbol{e}_{\nu}(\boldsymbol{r}) \frac{\cos \beta_{\nu} z_{2}}{\cos \beta_{\nu} g / 2},
$$

where $z_{2}=0$ is now the center of the iris region, and where we use Greek subscripts $(\nu, \mu, \sigma, \cdots)$ for the iris region. Equation (2) is appropriate for the modes in our symmetric structure for which $\boldsymbol{E}_{\perp}^{(I)}$ is even in $z_{2}$. For those modes where $\boldsymbol{E}_{\perp}^{(I)}$ is odd in $z_{2}$ we need to replace the cosines by sines in Eq. (2). We express the coef $囚$ cients $a_{n}$ and $b_{\nu}$ in terms of $\boldsymbol{E}_{\perp}(\boldsymbol{r}) \equiv \boldsymbol{u}(\boldsymbol{r})$ at the interface between the cavity and the iris $\left(z_{1}=L, z_{2}=-g / 2\right)$. We then write the transverse magnetic ${ }^{\circledR}$ eld in each region. Equating the transverse magnetic ${ }^{\circledR e l d ~ a t ~} z_{1}=L, z_{2}=-g / 2$ in the region $S_{1}$ leads to the integral equation for the unknown function $\boldsymbol{u}(\boldsymbol{r})$

$$
\int_{S_{1}} d S^{\prime} \boldsymbol{u}\left(\boldsymbol{r}^{\prime}\right) \cdot \stackrel{\leftrightarrow}{K}\left(\boldsymbol{r}, \boldsymbol{r}^{\prime}\right)=0
$$

where

$$
\begin{aligned}
\stackrel{\leftrightarrow}{K}\left(\boldsymbol{r}, \boldsymbol{r}^{\prime}\right) & =\sum_{n} \lambda_{n} \boldsymbol{e}_{n}(\boldsymbol{r}) \boldsymbol{e}_{n}\left(\boldsymbol{r}^{\prime}\right) \cot \beta_{n} L \\
& -\sum_{\nu} \lambda_{\nu} \boldsymbol{e}_{\nu}(\boldsymbol{r}) \boldsymbol{e}_{\nu}\left(\boldsymbol{r}^{\prime}\right) \tan \beta_{\nu} g / 2,
\end{aligned}
$$

and

$$
\begin{aligned}
& \lambda_{n}=\frac{Z_{0}}{Z_{n}}=\left\{\begin{array}{ll}
k / \beta_{n}, & T M \\
\beta_{n} / k, & T E
\end{array}\right\}, \\
& \lambda_{\nu}=\frac{Z_{0}}{Z_{\nu}}=\left\{\begin{array}{ll}
k / \beta_{\nu}, & T M \\
\beta_{\nu} / k, & T E
\end{array}\right\},
\end{aligned}
$$

with $Z_{0}=\sqrt{\mu_{0} / \epsilon_{0}}$ being the impedance of free space, $Z_{n}$ being the impedance of the ${ }^{\mathrm{a}}$ cavity $^{\mathrm{o}}$ wave guide and $Z_{\nu}$ is the impedance of the ${ }^{a}$ iris $^{\circ}$ waveguide.

By separating out the dominant term $N$ in the sum over $n$, one can cast Eq. (3) into a variational form for the frequency, with the trial function being $\boldsymbol{u}(\boldsymbol{r})$. The result is the implicit equation for the frequency

$$
\frac{\tan \beta_{N} L}{\lambda_{N}}=\sum_{\nu} \sum_{\mu} K_{N \nu}\left(M^{-1}\right)_{\nu \mu} K_{N \mu} .
$$


Here $M_{\nu \mu}$ is a symmetric matrix de®ned by

$$
\begin{aligned}
M_{\nu \mu}= & -\sum_{n \neq N}^{\prime} \lambda_{n} \cot \beta_{n} L K_{n \mu} K_{n \nu} \\
& +\sum_{\sigma} \lambda_{\sigma} \tan \beta_{\sigma g} g / 2 K_{\sigma F \mu} K_{\sigma \nu}
\end{aligned}
$$

and

$$
K_{n \nu} \equiv \int_{S_{1}} d S \boldsymbol{e}_{n} \cdot \boldsymbol{f}_{\nu}, K_{\sigma \nu} \equiv \int d S \boldsymbol{e}_{\sigma} \cdot \boldsymbol{f}_{\nu}
$$

Note that we are looking at modes close to the cavity modes corresponding to $\beta_{N} L=\ell \pi$ or $k_{N \ell}^{2}=\ell^{2} \pi^{2} / L^{2}+\gamma_{N}^{2}$. Since the frequency $k$ is also contained in $M_{\nu \mu}$, Eq. (6) must be solved by iteration.

We obtain the expression for the frequency change in the cavity and therefore generalize the concept of the static polarizability to a generalized polarizability by considering a mode which has a normal electric ${ }^{\circledR}$ eld, but no tangential magnetic ${ }^{\circledR}$ eld at the center of the hole (for example the $\mathrm{TM}_{0 \mathrm{~N} \ell}$ mode) and write

$$
\chi \equiv \frac{k^{2}-k_{M}^{2}}{k_{M}^{2} E_{M}^{2}(0)} .
$$

Similarly we obtain the generalization of the susceptibility for a circular hole (for example the $\mathrm{TM}_{1 \mathrm{~N} \ell}$ or $\mathrm{TE}_{1 \mathrm{~N} \ell}$ mode)

$$
\psi \equiv \frac{k_{M}^{2}-k^{2}}{k^{2} H_{M}^{2}(0)} .
$$

We obtain $k$ from Eq. (6) and $\chi$ or $\psi$, for that frequency, from Eq. (9) or (10). Clearly the mode identi®cation $M$ stands for $0 N \ell$ or $1 N \ell$ as appropriate.

\section{POLARIZABILITY FOR A CIRCULAR IRIS HOLE}

We now specialize to $\mathrm{TM}_{0 \mathrm{n}}$ waveguide modes in a circular geometry in order to obtain the polarizability[1]. The cavity radius is $b$ and the iris radius is $a$ and we use the complete set

$$
f_{\nu}(\boldsymbol{r})=\boldsymbol{e}_{\nu}(\boldsymbol{r})=-\nabla \phi_{\nu}(r)
$$

with

$$
\phi_{n}(r)=\frac{J_{0}\left(s_{n} r / b\right)}{\sqrt{\pi} s_{n} J_{1}\left(s_{n}\right)}, \phi_{\nu}(r)=\frac{J_{0}\left(s_{\nu} r / a\right)}{\sqrt{\pi} s_{\nu} J_{1}\left(s_{\nu}\right)} .
$$

Here $s_{n, \nu}$ are the roots of the equation $J_{0}\left(s_{n, \nu}\right)=0$. Evaluating the kernels and matrices in Eq. (8) and Eq. (7), the frequency can now be calculated using Eq. (6) and the symmetric polarizability using Eq. (9). Similarily we can obtain the asymmetric polarizability.

The polarizabilities obtained in this way will be functions of the geometrical parameters $a / b, g / a, a / L$, and $\ell, N$ of the $\mathrm{TM}_{\mathrm{ON} \ell}$ cavity mode. In order to tie the polarizabilities to the geometry of the hole alone, it is necessary to take the limit for large $b$ and $L$, but with ${ }^{\circledR}$ nite frequency. This can be accomplished by letting $b, L \rightarrow \infty$, but keeping $s \equiv s_{N} a / b \quad t \equiv \ell \pi a / L$ ®nite by also allowing $N, \ell \rightarrow \infty$.

\section{SUSCEPTIBILITY FOR A CIRCULAR HOLE}

We now must use the waveguide modes $\mathrm{TM}_{1 \mathrm{n}}$ and $\mathrm{TE}_{1 \mathrm{n}}$ for our complete set in the pipe region. Speci®cally we have

$$
\begin{gathered}
\boldsymbol{e}_{n}=-\boldsymbol{\nabla} \phi_{n}, \\
\phi_{n}=\sqrt{\frac{2}{\pi}} \frac{J_{1}\left(p_{n} r / b\right) \cos \theta}{p_{n} J_{0}\left(p_{n}\right)} \text { for } \mathrm{TM}_{1 \mathrm{n}} \text { modes, } \\
\boldsymbol{e}_{n}=\hat{\boldsymbol{z}} \times \boldsymbol{\nabla} \psi_{n}, \\
\psi_{n}=\sqrt{\frac{2}{\pi}} \frac{J_{1}\left(q_{n} r / b\right) \sin \theta}{\sqrt{q_{n}^{2}-1} J_{1}\left(q_{n}\right)} \text { for } \mathrm{TE}_{1 \mathrm{n}} \text { modes, }
\end{gathered}
$$

where $p_{n, \nu}$ are the roots of $J_{1}\left(p_{n, \nu}\right)=0$. In the iris region

$$
\begin{gathered}
\boldsymbol{e}_{\nu}=-\nabla \phi_{\nu} \\
\phi_{\nu}=\sqrt{\frac{2}{\pi}} \frac{J_{1}\left(p_{\nu} r / a\right) \cos \theta}{p_{\nu} J_{0}\left(p_{\nu}\right)} \text { for } \mathrm{TM}_{1 \nu} \text { modes }, \\
\boldsymbol{e}_{\nu}=\hat{\boldsymbol{z}} \times \nabla \psi_{\nu}, \\
\psi_{\nu}=\sqrt{\frac{2}{\pi}} \frac{J_{1}\left(q_{\nu} r / a\right) \sin \theta}{\sqrt{q_{\nu}^{2}-1} J_{1}\left(q_{\nu}\right)} \text { for } \mathrm{TE}_{1 \nu} \text { modes }
\end{gathered}
$$

where $q_{n, \nu}$ are the roots of $J_{1}^{\prime}\left(q_{n, \nu}\right)=0$. Once again, the kernels and matrix elements can be calculated, but now $n$ and $\nu$ can be either TM or TE in $K_{n \nu}^{\psi}$, where the superscript $\psi$ denotes susceptibility. The frequency is again obtained from Eq. (6), but the susceptibility requires using Eq. (10).

\section{DISCUSSION OF THE ANALYTIC RESULTS}

The variational formulation also allows us to obtain analytically the ${ }^{\circledR}$ rst order correction in $k^{2} a^{2}$ for a hole in a plate of zero thickness from the knowledge of the correct $® e l d$ solutions for $k a=0$. In the case of polarizbility we obtain the following approximate analytic form

$$
\chi_{N \ell} \cong \frac{4 a^{3}}{3}\left(1-\frac{k^{2} a^{2}}{5}-\frac{s^{2}}{5}+\frac{4 j}{9 \pi} k^{3} a^{3}\right) .
$$

In the case of susceptibility we obtain

$$
\begin{aligned}
\psi_{N \ell}^{T M} & \cong \frac{8 a^{3}}{3}\left[1+k^{2} a^{2}\left(\frac{8}{15}-\frac{p^{2}}{3 k^{2} a^{2}}-\frac{p^{4}}{15 k^{4} a^{4}}\right)\right. \\
& \left.-\frac{8 j}{9 \pi} k^{3} a^{3}\right], \\
\psi_{N \ell}^{T E} \cong & \frac{8 a^{3}}{3}\left[1+k^{2} a^{2}\left(\frac{8}{15}-\frac{q^{2}}{5 k^{2} a^{2}}\right)-\frac{8 j}{9 \pi} k^{3} a^{3}\right],
\end{aligned}
$$

where $p \equiv p_{N} a / b, q=q_{N} a / b$. It appears that the approximate forms for polarizability and susceptibility give reasonably accurate results even for values of $k a$ as large as 1 .

The coef®cients of the leading terms in Eqs. (17)-(19) for $g=0$ and small $k a$ appear to be well con ${ }^{\circledR}$ rmed. Moreover, the coef $₫$ cients of the terms in $\AA$ rst order in $k^{2} a^{2}$ and the coef®cients of the leading imaginary terms are the same as those obtained by Eggimann[2] . 


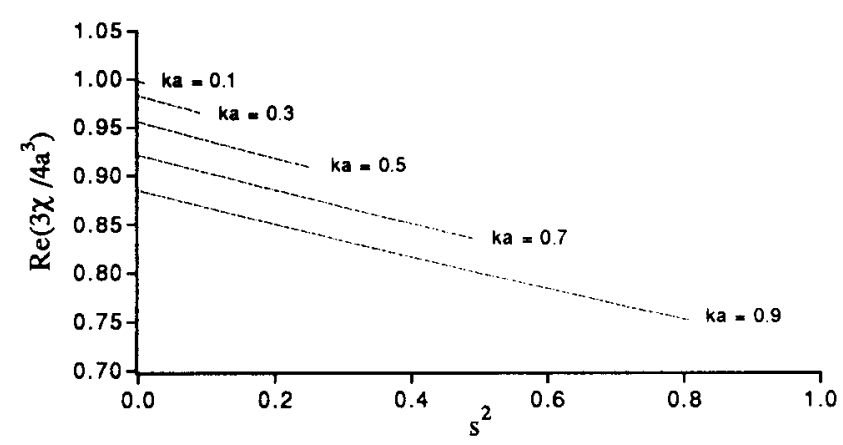

Figure. 1. Real part of scaled polarizability $\tilde{\chi}$ vs. $s^{2}$ for various $k a$, with $g / a=0$.

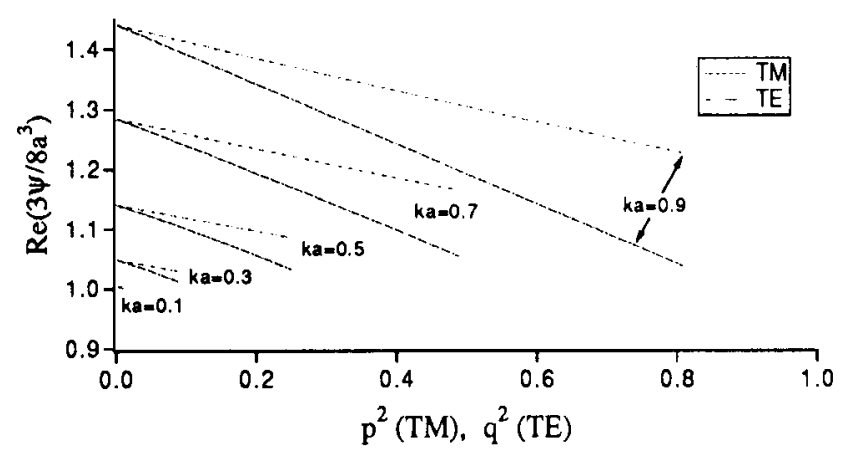

Figure. 2. Real part of scaled susceptibility $\tilde{\psi}$ vs. $p^{2}$ (for TM mode) or $q^{2}$ (for TE mode) for various $k a$, with $g / a=0$.

\section{NUMERICAL RESULTS FOR A HOLE IN A PLATE}

We present the results in a form suggested by the predictions for $g=0$ (zero wall thickness) and small $k a, s$ in Eq. (17). Speci®cally, we de®ne $\chi(k a, s) \equiv \chi /\left(4 a^{3} / 3\right)$ and plot the real part of $\tilde{\chi}$ vs. $s^{2}$ for various values of $k a$ in Fig. 1. A similar presentation is provided for the real part of the susceptibilities. We $\operatorname{de} \AA$ ne $\tilde{\psi} \equiv \psi /\left(8 a^{3} / 3\right)$ and plot $\tilde{\psi}$ vs. $p^{2}$ (TM) or $q^{2}$ (TE) for various values of $k a$ in Fig. 2.

For the wall with @nite thickness, the polarizability and susceptibility seen within the cavity are given by[1] $\chi_{i n}=\chi_{s}+$ $\chi_{a}, \psi_{i n}=\psi_{s}+\psi_{a}$, while the polarizability and susceptibility seen outside the cavity are given by $\chi_{\text {out }}=\chi_{s}-\chi_{a}, \psi_{\text {out }}=$ $\psi_{s}-\psi_{a}$. Here the subscripts $s$ and $a$ denote the solutions of the symmetric and antisymmetric potential problems[1] . In Figs. 3 and 4, we show $\tilde{\chi}_{i n}$ and $\tilde{\psi}_{i n}$ as functions of $g / a$ for various $k a$.

The logarithmic plots of $\tilde{\chi}_{\text {out }}$ and $\tilde{\psi}_{\text {out }}$ become linear with slopes $-s_{1}=-2.405$ and $-q_{1}=-1.841$, respectively, as expected.

\section{SUMMARY}

We have de®ned a generalized polarizability and susceptibility of a hole for ${ }^{\circledR}$ nite wave length in terms of the frequency shift of the associated cavities due to the hole. In addition we have constructed a variational form for these frequency shifts, assur-

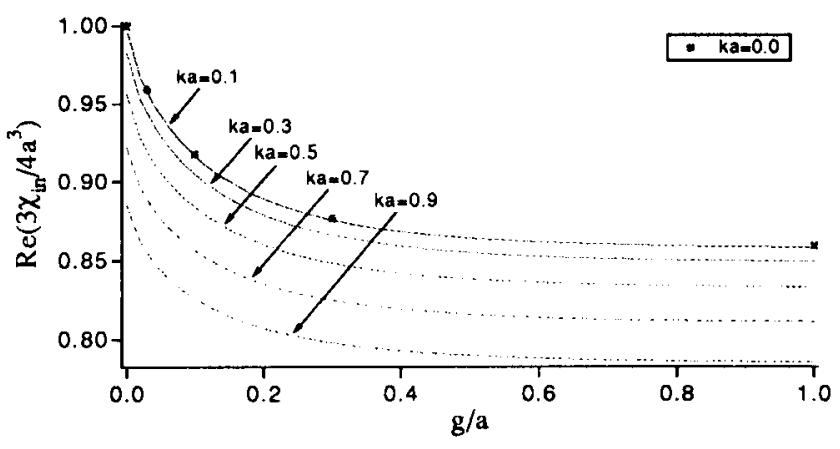

Figure. 3. Real part of scaled polarizability $\tilde{\chi_{i n}}$ vs. $g / a$ for various $k a$, with $s=0$.

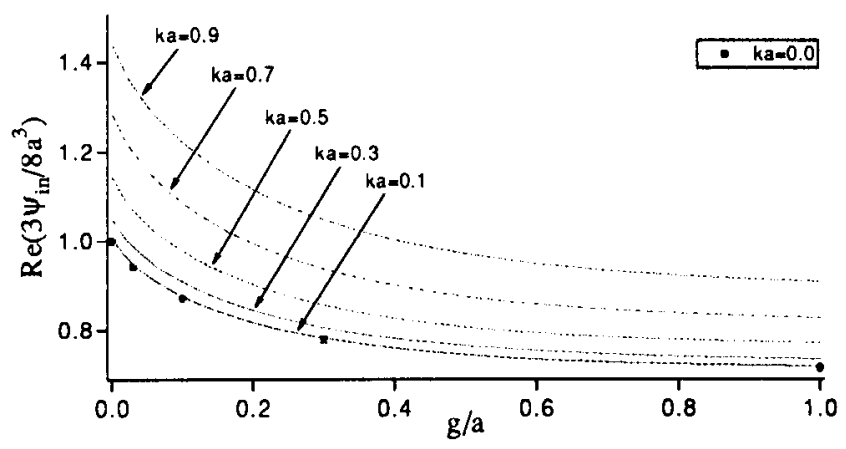

Figure. 4. Real part of scaled susceptibility $\tilde{\psi_{i n}}$ vs. $g / a$ for various $k a$, with $p=0$ (for TM mode) or $q=0$ (for TE mode), where the curves are the same for both TM and TE modes.

ing good convergence for our numerical calculations. We then allow the cavity dimensions to be in ${ }$ nitely large, enabling us to obtain accurate numerical values of the polarizability and susceptibilities of a circular hole in an in $₫$ nite plate of $®$ nite thickness. Then we obtain numerical results for various values of $k a$, $g / a$. The approximate analytic forms are given by Eq. (17) for the polarizability and by Eqs. (18), (19) for the susceptibility.

\section{References}

[1] The analysis for a circular hole in a thick wall follows that for $k=0$ in R.L. Gluckstern and J.A. Diamond, IEEE Transactions in Microwave Theory and Techniques, Vo. 39, No. 2, February 1991, p. 274, which contains references to earlier work.

[2] W.H. Eggimann, Higher Order Evaluation of Electromagnetic Diffraction by Circular Disks, IRE Transactions of Microwave Theory and Techniques, vol. MMT-9, pp. 408-418, September 1961. 